\title{
Co-Occurrence of High Degree Atrioventricular Block and Facial Pain in Lateral Medullary Stroke
}

\author{
Shairin Sihabdeen ${ }^{1}$, Elisa Ventura ${ }^{2}$, Carlo W Cereda ${ }^{1}$, Claudio Staedler ${ }^{1}$, Giacomo Chiaro ${ }^{1,3}$ and Mauro Manconi ${ }^{3,4,5 *}$ \\ ${ }^{1}$ Department of Neurology and Stroke Center, Neurocenter of Southern Switzerland, Lugano, Switzerland \\ ${ }^{2}$ Department of Neuroradiology, Neurocenter of Southern Switzerland, Lugano, Switzerland
}

${ }^{3}$ Sleep Center, Neurocenter of Southern Switzerland, Lugano, Switzerland

${ }^{4}$ Faculty of Biomedical Sciences, Università della Svizzera Italiana, Lugano, Switzerland

${ }^{5}$ Department of Neurology, University Hospital Inselspital, Bern, Switzerland

*Corresponding author: Mauro Manconi, Sleep Medicine, Neuro-center of Southern Switzerland, Regional Hospital of Lugano, Via Tesserete 46, 6903 Lugano, Switzerland

\begin{abstract}
Background: Besides the typical alternate clinical picture, Wallenberg syndrome may be rarely associated with facial pain due to the involvement of the trigeminal nucleus, and sudden death.

Case: We report a case of lateral medullary infarction associated with potentially life threatening intermittent high degree atrioventricular block which occurred in close temporally association with severe facial pain exacerbations. The case required an urgent implantation of cardiac pacing. We hypothesized that the AV-block was triggered by vasovagal response to centrally-mediated pain. However, a direct involvement of the autonomic brainstem network (solitary tract nucleus and dorsal motor nucleus) could not be excluded.

Conclusion: A similar sequence of events might explain at least part of the unexplained sudden deaths that seldom accompany Wallenberg syndrome. This report highlights the importance of continuous cardiac monitoring, in the acute phase of lateral bulbar ischemia, in order to prevent unfavorable outcome.
\end{abstract}

Keywords: Wallenberg Syndrome; Facial Pain; Trigeminal Nucleus; Atrio-Ventricular Block; Cardiac Arrythmia; Vaso-Vagal Response

\section{Introduction}

Wallenberg syndrome is the most frequent clinical manifestation of brainstem ischemia, and it is due to lateral medullary infarction consequential to the occlusion of the posterior inferior cerebellar artery or vertebral artery. The typical alternate clinical picture includes: vertigo with nystagmus and nausea (inferior vestibular nucleus); dysphonia, dysarthria, and dysphagia (nucleus ambiguous, NA); Horner syndrome (sympathetic fibers); ipsilateral limbs ataxia (restiform body, spinocerebellar fibers, inferior cerebellar peduncle); impaired ipsilateral facial sensory perception (trigeminal nucleus); pain and temperature perception impairment in the contralateral limbs (spinothalamic tract) and, when there is damage of the ventral medulla, minimal weakness of the contralateral limbs (cortico-spinal tract) [1].

However, depending on the topography of the lesion, other peculiar symptoms might be associated with lateral bulbar infarction. Among these, ipsilateral facial pain, which often recalls 
trigeminal neuralgia, is well described in the literature [2-5]. Although infrequent, sudden death might occur in Wallenberg syndrome, representing the most serious accompanying event $[6,7]$.

\section{Case Report}
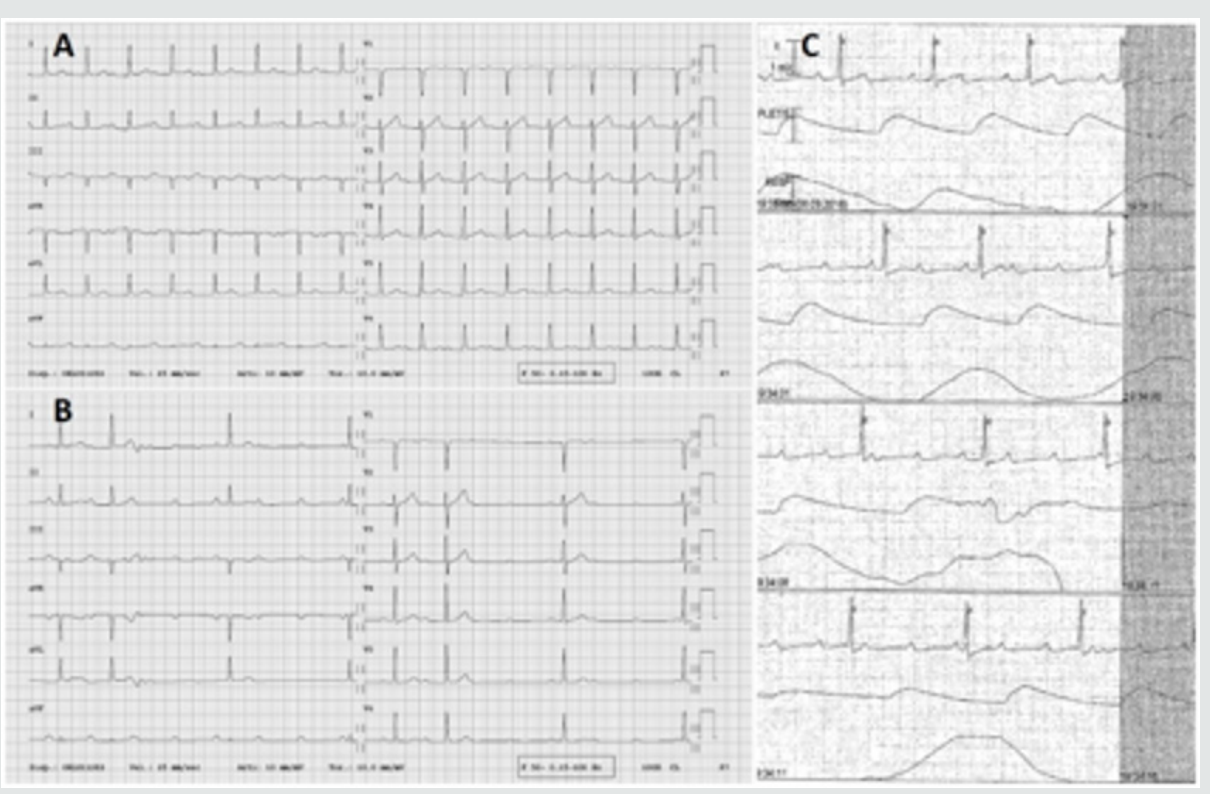

Figure 1: EKG pattern recorded during the acute phase of bulbar stroke in three different conditions. (A) First- degree atrioventricular conduction block during asymptomatic (inter-critical) basal recording; (B) third-degree atrioventricular conduction block during one episode of facial pain (critical); (C) transition from a first-degree atrioventricular conduction block to a third-degree atrioventricular conduction block in coincidence with a sudden severe facial pain exacerbation.

A 42-years-old overweight and smoking woman, with untreated hypertension, referred to the emergency room for acute and severe pain in her left ear, which rapidly extended to the left side of face. Symptoms were accompanied by dizziness and mild hypoesthesia of the left arm. Facial pain fluctuated in its severity, with the highest peaks accompanied by bradycardia of up to $33 \mathrm{bpm}$ and presyncopal symptoms. Simultaneous ECG monitoring showed a new seconddegree atrioventricular (AV) block, which, in coincidence with pain exacerbations, alternated with a transient third-degree AV block (Figure 1). These arrhythmias required the urgent positioning of a temporary external pacemaker.

Cardiac ultrasound, coronary angiography, hematological tests and brain angio-CT were normal. One day later, a brain diffusion magnetic resonance imaging (MRI) disclosed an acute ischemic
Herein, we describe and speculate on the pathogenesis of a peculiar case of Wallenberg syndrome, in which fluctuating severe facial pain and high degree atrioventricular block co-occur with a close temporal relationship. 

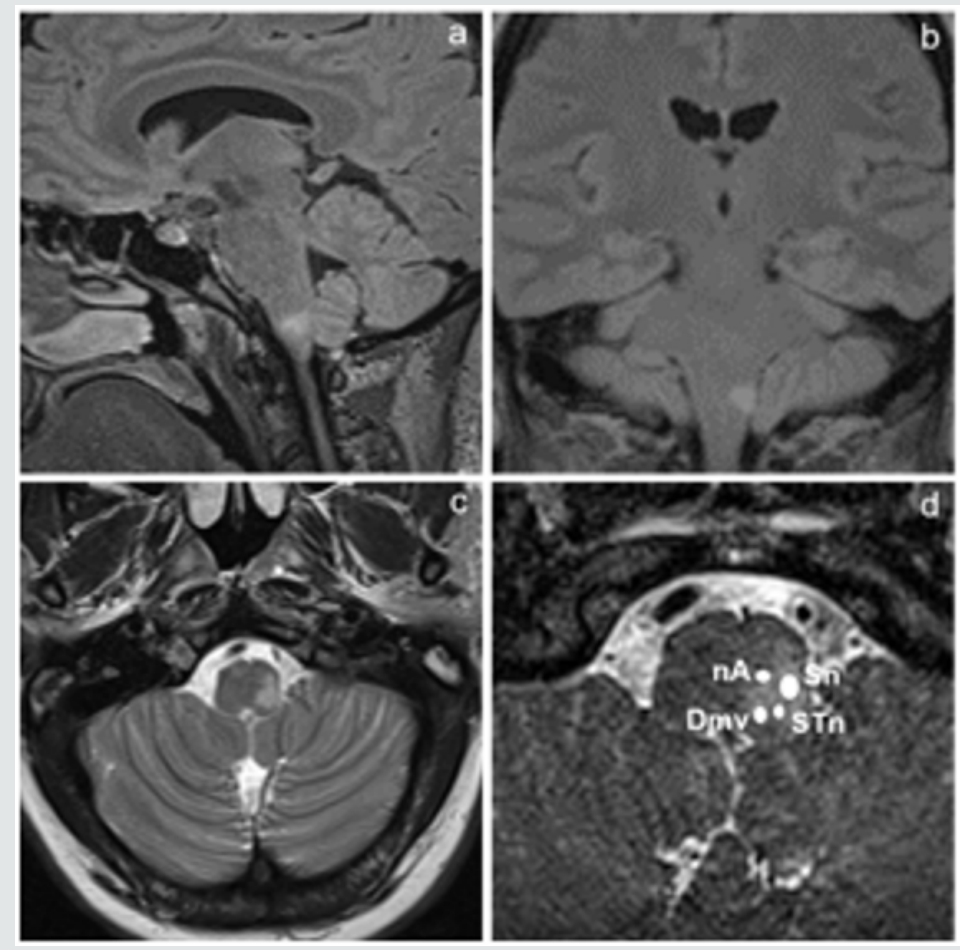

Figure 2: Sagittal and coronal T2-FLAIR (a, b) and axial T2 TSE (c) images demonstrate a focal hyperintense ischemic lesion in the left dorso-lateral aspect of medulla (vascular territory of the postero-inferior cerebellar artery). A 3D high-resolution T2SPACE axial image (d) shows the anatomical location of the left $X$ cranial nerve nuclei (Dmv: dorsal motor nucleus of vagus nerve, STn: solitary tract nucleus, nA: nucleus ambiguus) and the V cranial nerve spinal nucleus (Sn) within the medulla.

\section{Discussion}

To the best of our knowledge, this is the first observation of Wallenberg syndrome associated with acute fluctuating highdegree AV block occurring in coincidence with severe stabbing facial pain. The clinical picture and the MRI, recapitulate the lateral bulbar syndrome, with involvement of: lower parts (pars caudalis and interpolaris) of the trigeminal nucleus (ipsilateral lower face pain); restiform nucleus (ipsilateral ataxia); medial lemniscus tract (ipsilateral upper limb mild hypoesthesia); vestibular nucleus (dizziness); cortico-spinal tract (ipsilateral mild upper limb palsy); and spinothalamic tract (contralateral limbs and body pain and impairment of temperature sensitivity) [8].

The acute pain, initially reported by the patient in her ear, is in line with spinal trigeminal nucleus receiving pain input from the ear pinna. The basis of the fluctuating AV block is more uncertain, with two possible mechanisms explaining its origin. Firstly, the stroke involved the lower trigeminal spinal nucleus, causing intense ipsilateral facial pain, which in turn favored the AV block by means of a vasovagal response. Secondly, the ischemic lesion involved autonomic brainstem networks (IX and X cranial nerves), such as the solitary tract nucleus (STN) and/or the dorsal motor nucleus of the vagus nerve (DMV), with the first collecting autonomic visceral afferent from the carotid body, carotid sinus, aortic bodies and sinoatrial node, and the second one projecting efferent to the heart. A further, albeit less probable mechanism, might involve the trigemino-cardiac reflex, which consists in a reflexive response to mechanical, electrical or chemical stimulation of the trigeminal nerve and/or ganglion or brainstem centers during surgery/angiography, leading to severe dysrhythmias and arterial hypotension $[9,10]$. Such reflex, however, is usually mediated by an external stimulus, which is missing in the present case.

Our first hypothesis (vasovagal response to pain) is supported by the tight temporal co-occurrence between stabbing pain exacerbations and AV blocks (Figure 1). Fibers from the trigeminal nerve have been shown to project to the STN and some authors described a connection between the trigeminal ganglion and the vagus nerve $[9,11,12]$. Moreover, against the second hypothesis (direct involvement of STN) stands the absence of gustatory deficits, which indicates a possible sparing of the STN.

On the other hand, based on MRI scan, the lesion seemed to involve both medial and lateral medulla areas in its posterior part, while only touching the lateral area in its frontal part. These findings support our second hypothesis (direct autonomic impairment) of a direct involvement of both STN and DVM. The nucleus ambiguous 
(antero-medial medulla area) was spared, and this could explain the absence of bulbar motor symptoms (dysarthria, dysphagia and dysphonia) (Figure $2 \mathrm{c}, \mathrm{d}$ ).

Trigeminal neuralgia secondary to lateral medullary infarction is a well-described rarity [2-5]. Vagal-mediated first- and seconddegree AV blocks have been observed [13], while a third-degree AV block secondary to a pain-related vagal activation has never been described. Koay et al. documented a case with several asymptomatic sinus arrests in a patient with left lateral medulla infarction. As well as in our case, the authors did not find any clear cardiac disease, and treated the patient with a permanent PM [6].

The present observations yield three interesting speculations:

1) At least part of the sudden deaths described in Wallenberg syndrome might indeed be due to a non-documented high-degree $\mathrm{AV}$ block. Even in our case, a delay of pacing intervention could have had lethal consequence;

2) Occurrence of intense facial pain possibly favors vasovagal responses with variable impairment of $\mathrm{AV}$ conduction;

3) Previous cases of sudden deaths in Wallenberg syndrome should be revisited in order to check for co-occurrence of severe facial pain. Based on these speculations, we recommend to monitor ECG already in ER in all cases of lateral medulla syndrome, being prepared for possible heart pacing, especially in cases accompanied by facial pain.

\section{Conflicts of Interests}

The authors certify that they have no affiliations with or involvement in any organization or entity with any financial interest (such as honoraria; educational grants; participation in speakers' bureaus; membership, employment, consultancies, stock ownership, or other equity interest; and expert testimony or patentlicensing arrangements), or non-financial interest (such as personal or professional relationships, affiliations, knowledge or beliefs) in the subject matter or materials discussed in this manuscript.

\section{References}

1. Albert F Peterman, Robert G Siekert (1960) The Lateral Medullary (Wallenberg) Syndrome: Clinical Features and Prognosis. Medical Clinics of North America 44(4): 887-896.

2. Carlos M Ordás, María L Cuadrado, Patricia Simal, Raúl Barahona, Javier Casas, et al. (2011) Wallenberg's syndrome and symptomatic trigeminal neuralgia. J Headache Pain 12(3): 377-380.

3. Abinayaa Ravichandran, Kareem S Elsayed, Hussam A Yacoub (2019) Central Pain Mimicking Trigeminal Neuralgia as a Result of Lateral Medullary Ischemic Stroke. Case Reports in Neurological Medicine Pp: $1-3$.

4. Jong Sung Kim (1993) Trigeminal Sensory Symptoms due to midbrain lesion. Eur Neurol 33(3): 218-220.

5. D Canovas, J Vinas, A Rovira (2004) Lateral bulbar infarction with involvement of the trigeminal spinal nucleus. Neurologia 19(1): 23.

6. Shiwen Koay, Bikash Dewan (2013) An unexpected Holter monitor result: multiple sinus arrests in a patient with lateral medullary syndrome. BMJ Case rep 2013:bcr2012007783.

7. Jaster JH, Porterfield LM, Bertorini TE, Dohan FC Jr, Becske T (1995) Cardiac arrest following vertebrobasilar stroke. J Tenn Med Assoc 88(8): 309.

8. Jong S Kim (2003) Pure lateral medullary infarction: clinical-radiological correlation of 130 acute, consecutive patients. Brain 126(Pt 8): 18641872.

9. David F Bauer, Andrew Youkilis, Christine Schenck, Christopher R Turner, B Gregory Thompson (2005) The falcine trigeminocardiac reflex: case report and review of literature. Surgical Neurology 63(2): 143-148.

10. Takamitsu Tamura, David E Rex, Miklos G Marosfoi, Ajit S Puri, Matthew J Gounis, et al. (2016) Trigeminocardiac reflex caused by selective angiography of the middle meningeal artery. J Neurointerv Surg 9(3): e10.

11. Kumada M, Dampney RA, Reis DJ (1977) The trigeminal depressor response: a novel vasodepressor response originating from the trigeminal system. Brain research 119(2): 305-326.

12. Reiji Kishida, Tjard de Cock Buning, Jacob L Dubbeldam (1983) Primary vagal nerve projections to the lateral descending trigeminal nucleus in boidae (Python molurus and Boa constrictor). Brain Res 263: 132-136.

13. Paolo Alboni, Anna Holz, Michele Brignole (2013) Vagally mediated atrioventricular block: pathophysiology and diagnosis. Heart 99(13): 904-908.
To Submit Your Article Click Here:

This work is licensed under Creative Commons Attribution 4.0 License

DOI: $10.32474 / 0 J N B D .2020 .04 .000195$

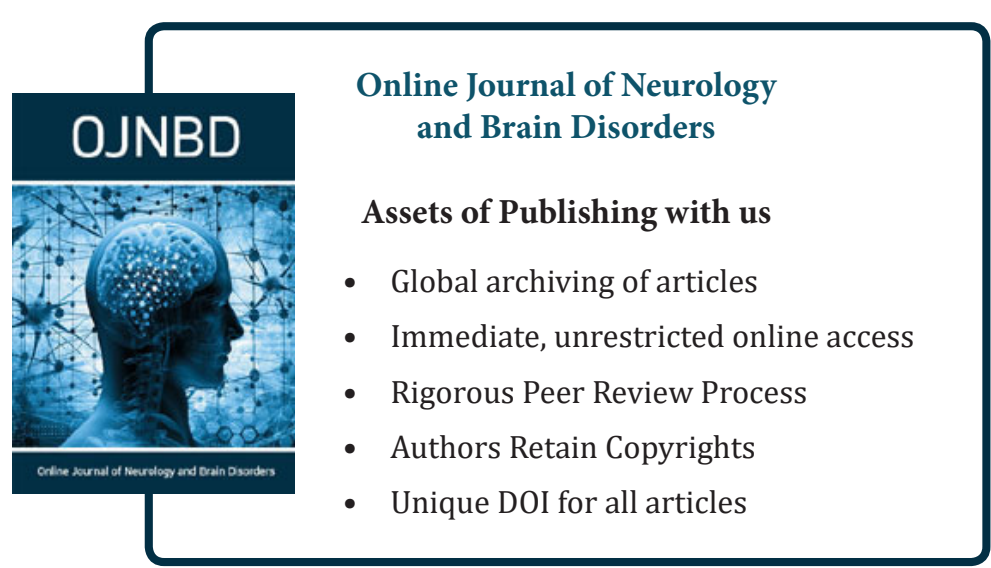

